\title{
Sensing Power Transfer Between the Human Body and the Environment
}

\author{
Peter H. Veltink*, Senior Member, IEEE, Henk Kortier, and H. Martin Schepers, Student Member, IEEE
}

\begin{abstract}
The power transferred between the human body and the environment at any time and the work performed are important quantities to be estimated when evaluating and optimizing the physical interaction between the human body and the environment in sports, physical labor, and rehabilitation. It is the objective of the current paper to present a concept for estimating power transfer between the human body and the environment during free motions and using sensors at the interface, not requiring measurement systems in the environment, and to experimentally demonstrate this principle. Mass and spring loads were moved by hand over a fixed height difference via varying free movement trajectories. Kinematic and kinetic quantities were measured in the handle between the hand and the load. 3-D force and moments were measured using a 6 DOF force/moment sensor module, 3-D movement was measured using 3-D accelerometers and angular velocity sensors. The orientation was estimated from the angular velocity, using the initial orientation as a begin condition. The accelerometer signals were expressed in global coordinates using this orientation information. Velocity was estimated by integrating acceleration in global coordinates, obtained by adding gravitational acceleration to the accelerometer signals. Zero start and end velocities were used as begin and end conditions. Power was calculated as the sum of the inner products of velocity and force and of angular velocity and moment, and work was estimated by integrating power over time. The estimated performed work was compared to the potential energy difference corresponding to the change in height of the loads and appeared to be accurate within $4 \%$ for varying movements with net displacements and varying loads (mass and spring). The principle of estimating power transfer demonstrated in this paper can be used in future interfaces between the human body and the environment instrumented with body-mounted miniature 3-D force and acceleration sensors.
\end{abstract}

Index Terms-Ambulatory sensing, force sensing, inertial movement sensing, power estimation, work estimation.

\section{INTRODUCTION}

$\mathbf{T}$ HE PHYSICAL interaction between the human body and the environment is important in many situations: In physical labor, interactions with the environment need to be per-

Manuscript received October 26, 2008; revised January 13, 2009. First published February 20, 2009; current version published June 10, 2009. This work is supported by the Dutch Technology Foundation STW, applied science division of NWO and the Technology Program of the Ministry of Economic Affairs. Asterisk indicates corresponding author.

${ }^{*} \mathrm{P}$. H. Veltink is with the Institute for Biomedical Technology (BMTI) and the Faculty of Electrical Engineering, Mathematics and Computer Science, University of Twente, $7500 \mathrm{AE}$ Enschede, The Netherlands (e-mail: p.h.veltink@utwente.nl).

H. Kortier and H. M. Schepers are with the Institute for Biomedical Technology (BMTI) and the Faculty of Electrical Engineering, Mathematics and Computer Science, University of Twente, 7500 AE Enschede, The Netherlands (e-mail: h.g.kortier@student.utwente.nl; h.m.schepers@utwente.nl).

Color versions of one or more of the figures in this paper are available online at http://ieeexplore.ieee.org.

Digital Object Identifier 10.1109/TBME.2009.2014963 formed within safe limits of body loading [1]-[3]. In rehabilitation, people need to relearn functional motor tasks [4]-[6] and interact with mobility support devices like wheelchairs or hand-cycling units [7], [8]. In sports, motor tasks are trained to the ultimate, maximizing force and/or endurance and optimizing coordination [9]-[11]. Robots become more and more versatile, performing varying tasks in physical interaction with the environment and with the human body, including training of upper and lower extremity motor tasks [12]-[15]. All of these examples involve the physical interaction between human body and environment. In all of these cases, this dynamic interaction is to be optimized or improved. For this purpose, it is essential to assess this interaction quantitatively in terms of force and movement at the interface, power transfer and timing, work performed, and effective dynamics of both bodies during the performance of functional tasks, preferably in the actual daily life settings. Such a quantitative assessment, possibly combined with electrophysiological measurements of muscle activation and biomechanical analysis, may result in a better functional understanding of the neuromuscular system under healthy and diseased conditions during realistic dynamic interactions encountered in daily life. It may add to new telemedicine approaches for continuous diagnosis and training of patients with neuromuscular disorders [16].

Until now, the dynamic analysis of human body movements has typically been performed by instrumenting the environment. Lumbar loading during lifting has been analyzed using force plates and optical 3-D movement analysis system in combination with biomechanical models [3], [17], [18]. More recently, a combination of electromyography (EMG), inertial sensor measurements of body movements and biomechanical modeling, was proposed for estimating lumbar loading, without actually measuring the mechanical interaction with the load or the ground [19]. Instrumented environments have also been used in studies of human performance in sports [10], [11] and physical rehabilitation training [7], [8], [13], [20], [21]. Only in restricted movements, power transfer between the human body and the environment has been estimated, for example, based on measured crank moment and pedal frequency during cycling [8] or using instrumented ergometers [11].

To our knowledge, power transfer and dynamic interaction between the human body and the environment during arbitrary free movements have not been assessed based on measurements at the moving interface between the human body and the environment. It seems very logical to do so when considering both interacting bodies as an interconnection of energy-exchanging subsystems [22]. The power transfer at any time instance can be quantified by multiplying effort and flow variables in the 
concerned physical domain. In the case of mechanical interaction between the human body and the environment, effort and flow variables are force and velocity. Sensing of both quantities would require full 3-D measurements of interaction force and velocity. The perpendicular component of the interaction force between body and environment is commonly measured in clinical research and applications using matrices of pressure sensitive resistors [23]. However, this approach does not provide full 3-D interaction force. Until now, no 3-D stress sensors have become available that could be adequately used at the interface between the human body and the environment, although several methods have been proposed, applying either piezoelectric or optical transduction methods [24], [25]. 3-D velocity can be adequately estimated from inertial and magnetic sensors placed on the human body. In recent years, many studies have developed methods to derive orientation, velocity, and change of position from such sensors [26]-[42]. Recently, we have proposed to combine inertial and force sensing in the analysis of the ground reaction forces during the stance and foot movements during the swing phase of gait [40], [43], [44]. It should be noted, however, that power transfer is not an issue under this condition, since no power is transferred when walking over a rigid floor.

It is the objective of the current paper to present a concept for estimating power transfer between the human body and the environment during free motions, using sensors on the interface, not requiring measurement systems in the environment, and to experimentally demonstrate this principle. A preliminary version of this work has been reported [45].

\section{MethodS}

\section{A. Estimation of Power and Work}

In the mechanical domain, power transfer $P$ between two bodies is given by

$$
P=\vec{F} \cdot \vec{v}+\vec{M} \cdot \vec{\omega}
$$

The first term represents the power transferred by displacement, equal to the inner product of force $\vec{F}$ and velocity $\vec{v}$ measured in the same coordinate system. The second term represents the power transferred by rotation, given by the inner product of moment $\vec{M}$ and angular velocity $\vec{\omega}$, also measured in the same coordinate system.

Moment and force can be measured using a 6 DOF force and moment sensor and angular velocity $\vec{\omega}$ using a $3-\mathrm{D}$ rate gyroscope. The essential difficulty is to estimate 3-D velocity during free motions using merely body-mounted sensors. We propose to estimate velocity from accelerometer measurements.

A 3-D accelerometer senses the difference between inertial and gravitational acceleration ( $\vec{a}$ and $\vec{g}$, respectively):

$$
\vec{s}_{a}=\vec{a}-\vec{g} .
$$

3-D velocity $\vec{v}$ can be obtained through integration of acceleration $\vec{a}$, which can be estimated from the accelerometer signals $\vec{s}_{a}$ by adding the gravitational acceleration $\vec{g}$. Since $\vec{g}$ is directed vertically and has a constant and known modulus on a certain place on earth (approximately $9.81 \mathrm{~m} / \mathrm{s}^{2}$ ), it can be added when the inclination of the sensor coordinate system (the orientation relative to the vertical) is known. Luinge and Veltink [33] have shown that inclination and accelerometer offset can be estimated from the 3-D accelerometer signals during human movements using an adequate Kalman filter, the inertial acceleration being modeled as a disturbance. The expected accuracy of this estimation depends on the size of this disturbance relative to the gravitational acceleration. When adding a 3-D angular velocity sensor (rate gyroscope), the full 3-D orientation can be estimated more accurately, leaving only drift around the vertical [34]. However, errors in the orientation estimation are still correlated with errors in the estimates of velocity and change of position, since the accelerometer as well as gyroscope signals are used in both estimates. For this reason, it is best to use gyroscope measurements only for orientation estimation and accelerometer measurements only for subsequent estimation of velocity and displacement [38], [46]. Due to the required integration, the orientation estimation obtained using gyroscopes is only accurate if the initial orientation is known and independent orientation information is available at regular periods of time [38], [46]. It should be noted that a similar requirement applies for estimating velocity and position from accelerometer measurements. This approach has been successfully applied in estimating orientation and relative positions combining inertial measurements with regular updates from magnetic actuation on the body [38] and for estimating foot movement during the swing phase of gait, applying known initial and final conditions during subsequent stance phases [43]. It is also the approach taken in the current study, assuming power transfer is to be estimated during relatively short interactions between body and environment, and initial and final conditions are given, e.g., initial and final velocities zero, and initial and final inclinations known or measured independently.

Thus, orientation is obtained by integration of angular velocity, solving the following differential equation [47]:

$$
\dot{R}_{S}^{G}=R_{S}^{G} \tilde{\omega}_{S, G}^{S} \cdot
$$

With $R_{S}^{G}$ being the sensor orientation in global coordinates, represented by a rotation matrix, expressing the sensor coordinate frame $\Psi_{S}$ in the global coordinate frame $\Psi_{G} \cdot \dot{R}_{S}^{G}$ is a matrix containing the time derivatives of all components of $R_{S}^{G}$, which can be integrated to find $R_{S}^{G}$ as a function of time. $\tilde{\omega}_{S, G}^{S}$ is the angular velocity measured by the rate gyroscope sensors, represented by a skew-symmetric matrix, expressing the angular velocity of frame $\Psi_{S}$ with respect to $\Psi_{G}$ in frame $\Psi_{S}$ :

$$
\tilde{\omega}_{S, G}^{S}=\left(\begin{array}{ccc}
0 & -\omega_{z} & \omega_{y} \\
\omega_{z} & 0 & -\omega_{x} \\
-\omega_{y} & \omega_{x} & 0
\end{array}\right)
$$

The global coordinate system $\Psi_{S}$ was defined to be identical to the sensor coordinate system $\Psi_{G}$ at start time $t_{0}$, the $z$-axis being vertical. This corresponds to the initial rotation matrix $R_{S}^{G}\left(t_{0}\right)$ being equal to the identity matrix, thus specifying the initial orientation required for solving differential equation (3). No assumption was made for the final orientation $R_{S}^{G}\left(t_{e}\right)$.

Using the estimated orientation as a function of time, the accelerometer signal $\vec{s}_{a}(t)$ is expressed in global coordinates. 
Velocity is subsequently estimated by integrating acceleration $\vec{a}^{G}$ after adding $\vec{g}^{G}$ to the accelerometer signal $\vec{s}_{a}^{G}(t)(2)$ :

$$
\begin{aligned}
\vec{a}^{G}(t) & =\vec{s}_{a}^{G}(t)+\vec{g}^{G} \\
\vec{v}^{-G}(t) & =\vec{v}^{G}\left(t_{0}\right)+\int_{t_{0}}^{t} \vec{a}^{G}(\tau) d \tau .
\end{aligned}
$$

This velocity estimate is corrected by applying information about end velocity $\vec{v}^{G}\left(t_{e}\right)$ :

$$
\vec{v}^{+G}(t)=\vec{v}^{-G}(t)+\frac{t-t_{0}}{t_{e}-t_{0}}\left(\vec{v}^{G}\left(t_{e}\right)-\vec{v}^{-G}\left(t_{e}\right)\right) .
$$

In the current study, begin and end velocities $\vec{v}^{G}\left(t_{0}\right)$ and $\vec{v}^{G}\left(t_{e}\right)$ were both equal to zero.

Finally, work performed can be estimated by integrating power over time:

$$
W(T)=\int_{0}^{T} P(t) d t .
$$

In order to calculate power according to (1), either velocity $\vec{v}(t)$, available in global coordinates, needs to be expressed in sensor coordinates or force $\vec{F}(t)$ is to be expressed in global coordinates using orientation estimates at any time $((3)$ and (4)). Moment $\vec{M}(t)$ and angular velocity $\vec{\omega}(t)$ are both readily available in sensor coordinates.

\section{B. Experimental Methods}

The feasibility of the proposed method of power and work estimation for relatively short interactions between the human body and a load was tested experimentally for mass and spring loads. The experimental setup is depicted in Fig. 1. It consisted of a handle, instrumented with a 3-D inertial and magnetic sensor unit (MTx, Xsens Motion Technologies) rigidly and closely connected to a 6 DOF force/moment sensor unit (ATI-Mini45SI-580-20, Schunk GmbH \& Co. KG). The acceleration range of the MTx sensor was $\pm 100 \mathrm{~m} / \mathrm{s}^{2}$, the angular velocity range was $\pm 1200^{\circ}$ s. The force sensing range of the ATI mini sensor was $\pm 580 \mathrm{~N}$ in $x$ - and $y$-directions and $\pm 1160 \mathrm{~N}$ in the axial $z$-direction. The moment ranges were $\pm 20 \mathrm{Nm}$ in all three directions. The force/moment sensor unit was mounted between handle and load, measuring the force and moment that both bodies exerted on each other at the interface.

The mass load had a mass of $9.37 \mathrm{~kg}$. It was repeatedly lifted from the ground onto a $0.75 \mathrm{~m}$ high table, accounting for a potential energy change of $68.9 \mathrm{~J}$. The movements were varied, allowing for minimal rotation of the mass during the transition (condition a), applying minimal moment to the handle and allowing pendulum-like movements during the transfer (condition b), and transfer under tilted condition (condition c). The horizontal displacement varied between 0.3 and $1.0 \mathrm{~m}$.

The spring load was an extension spring (Tevema T39210) with a spring constant of $87.9 \mathrm{~N} / \mathrm{m}$ and a zero force length of $1.0 \mathrm{~m}$. The lower end of the spring was attached to a vertical iron construction beam $0.15 \mathrm{~m}$ from the ground, the other side to the instrumented handle. The handle could be secured to either of two hooks fastened to the construction beam at different heights with respect to the ground: 1.85 and $2.34 \mathrm{~m}$. The handle was (a)

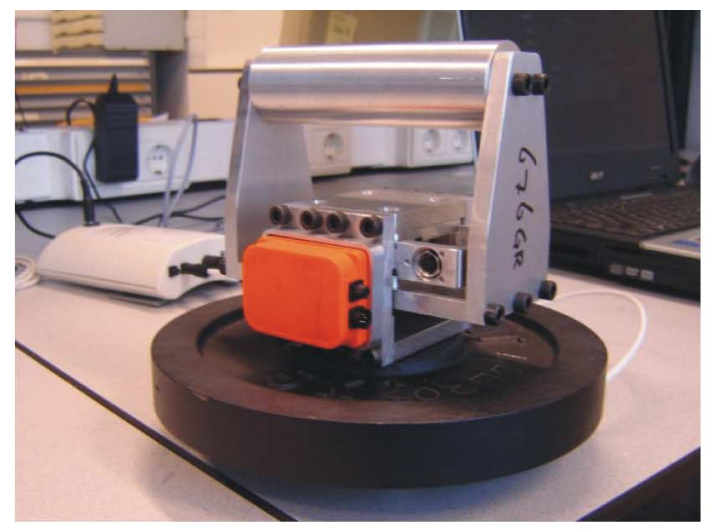

(b)
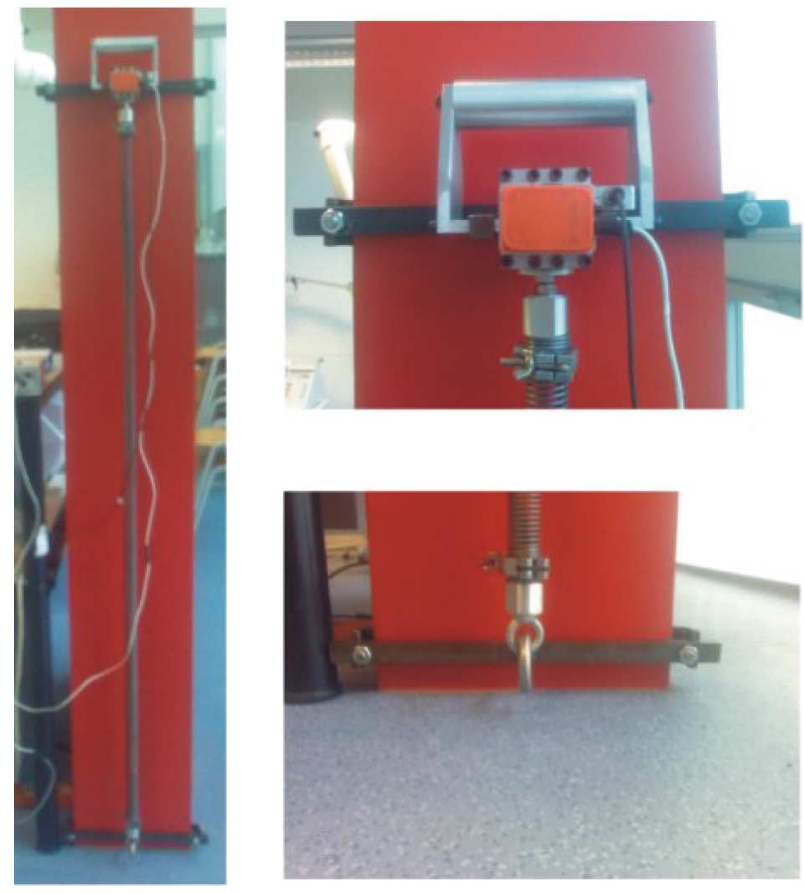

Fig. 1. Experimental setup: instrumented handle loaded by (a) a mass or (b) an extension spring.

repeatedly moved from the lower hook, extended, and secured to the upper hook (condition d), accounting for a potential energy difference of $10.1 \mathrm{~J}$. Condition a was repeated 19 times and conditions b-d were repeated 20 times.

\section{RESULTS}

Example results of the mass and spring movement trials are shown in Fig. 2(a) and (b), respectively. The presented trials were performed under conditions $b$ and $d$. For each condition, the sensor signals (acceleration, angular velocity, force, and moment), reconstructed linear and rotational movements, translational, rotational, and total power and work performed are presented. Under all conditions, the rms value of the rotational power was negligible in comparison to the rms value of the translational power (Table I).

The mean inclination was $0.06 \pm 0.01 \mathrm{rd}$ for condition a, $0.39 \pm 0.03 \mathrm{rd}$ for condition $\mathrm{b}$, and $0.33 \pm 0.09 \mathrm{rd}$ for condition 
(a)
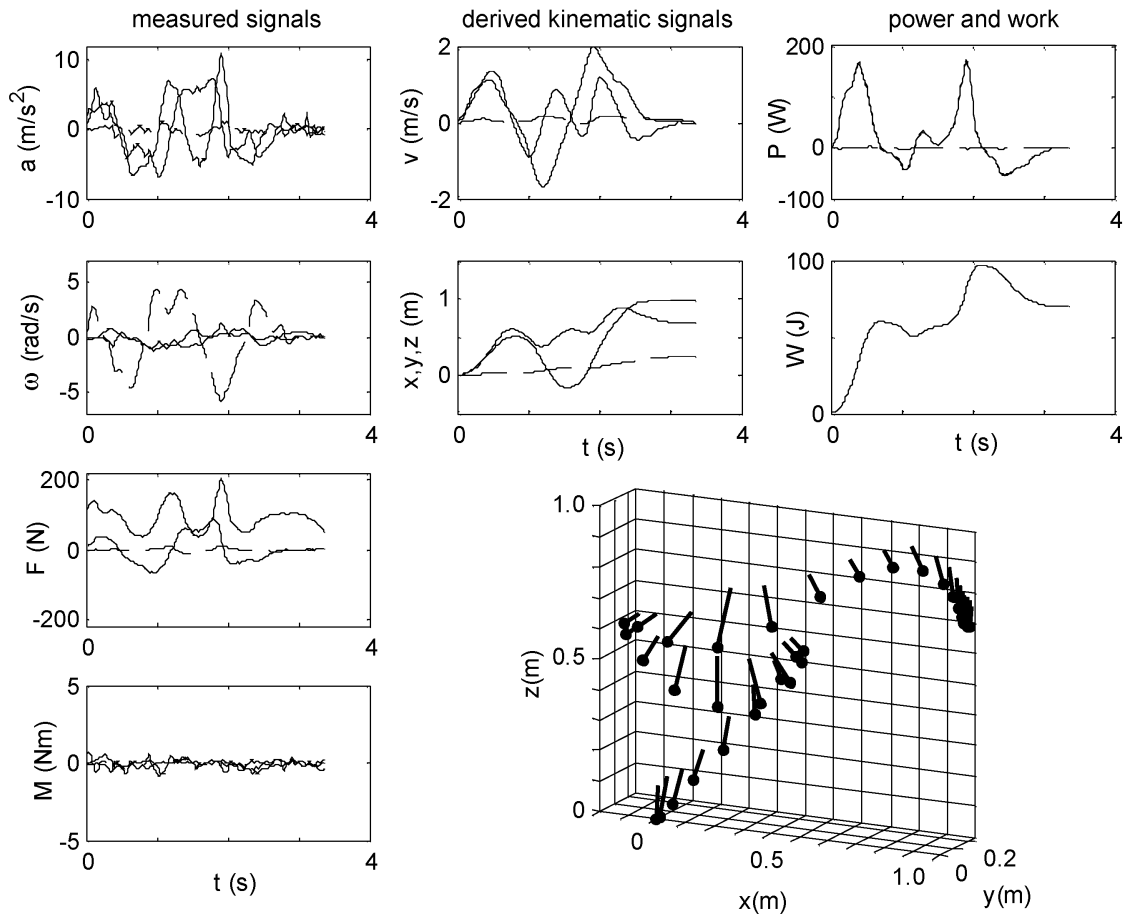

(b)
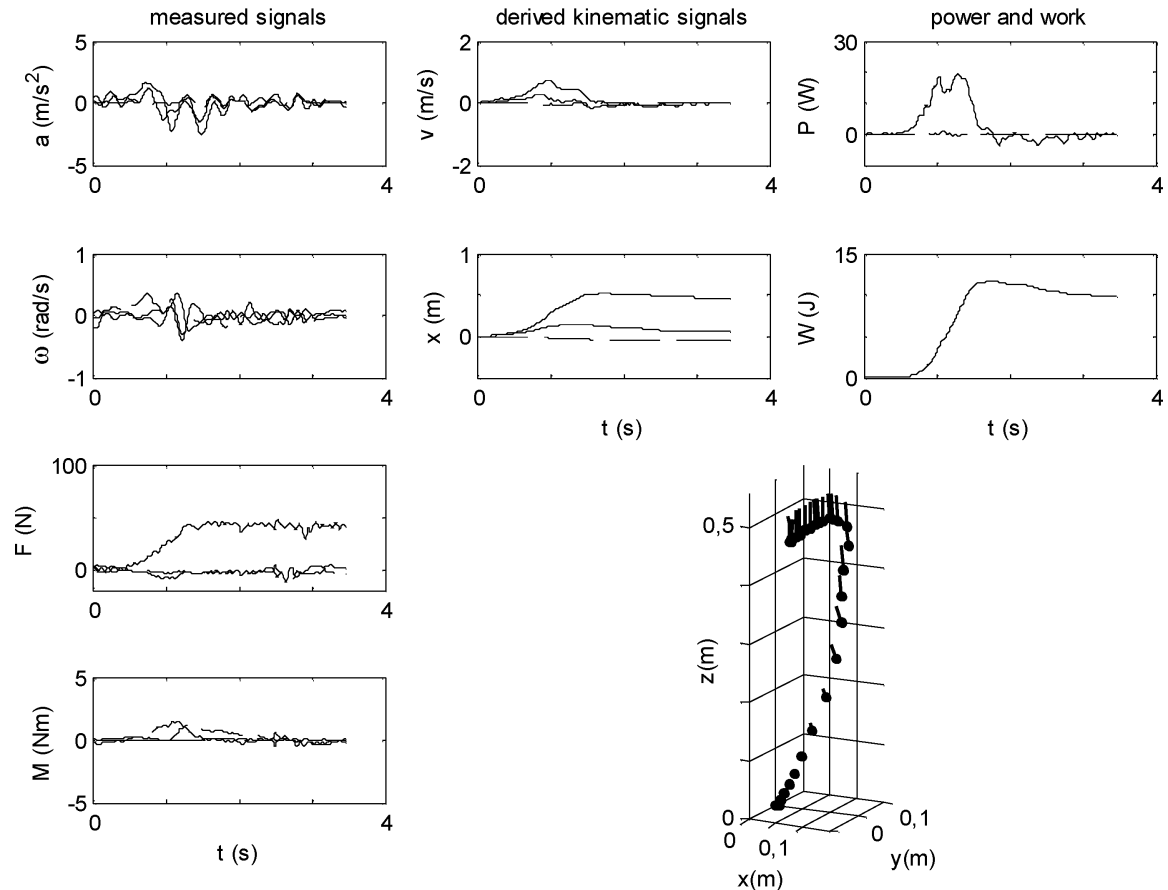

Fig. 2. Example measurement results for (a) a mass movement trial under condition b (mimal moment applied to the handle) and (b) a spring movement trial

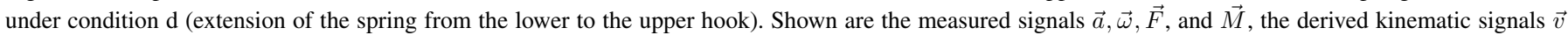

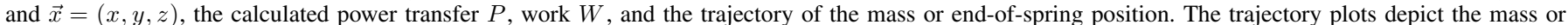

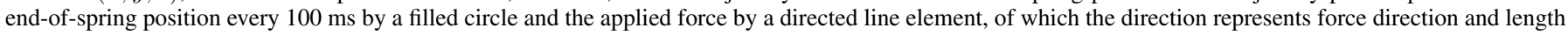
the size of the force. The $x$-components of the measured and derived kinematic signals are depicted by solid, $y$-components by long-dashed, and $z$-component by

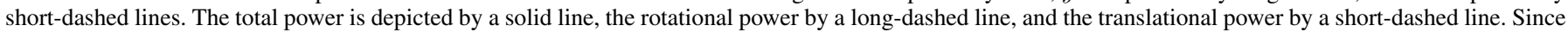
the rotational power is nearly zero, the translational power almost coincides with the rotational power under both conditions. 
TABLE I

TRANSLATiOnAl AND Rotational POWER EXERTED FOR THE MASS AND SPRING MOVEMENTS

\begin{tabular}{cccc}
\hline \hline Load & $\begin{array}{c}\text { Movement } \\
\text { condition }\end{array}$ & $\begin{array}{c}\text { RMS } \\
\text { translational } \\
\text { power (W) }\end{array}$ & $\begin{array}{c}\text { RMS } \\
\text { rotational } \\
\text { power (W) }\end{array}$ \\
\hline Mass & $\mathrm{a}$ & $40.5 \pm 4.3$ & $0.1 \pm 0.0$ \\
& $\mathrm{~b}$ & $55.4 \pm 7.1$ & $1.1 \pm 0.3$ \\
Spring & $\mathrm{c}$ & $27.3 \pm 3.7$ & $1.2 \pm 0.3$ \\
\hline \hline
\end{tabular}

TABLE II

ESTIMATED WORK AND HEIGHT DiFFERENCE FOR MOVING A MASS ONTO A TABLE AND LENGTHENING A SPRING

\begin{tabular}{ccccccc}
\hline \hline \multirow{2}{*}{ Load } & $\begin{array}{c}\text { Movement } \\
\text { condition }\end{array}$ & $\begin{array}{c}\text { Duration } \\
(\mathrm{s})\end{array}$ & \multicolumn{2}{c}{ Estimated work } & \multicolumn{2}{c}{ Estimated height } \\
& & $\mathrm{J})$ & Error $(\%)$ & $\left(* 10^{-2} \mathrm{~m}\right)$ & Error $(\%)$ \\
\hline \multirow{2}{*}{ Mass } & $\mathrm{a}$ & $3.3 \pm 0.3$ & $68.2 \pm 1.4$ & $-1.1 \pm 2.1$ & $75.8 \pm 1.0$ & $1.1 \pm 1.4$ \\
& $\mathrm{~b}$ & $3.5 \pm 0.2$ & $67.4 \pm 3.0$ & $-2.2 \pm 4.3$ & $69.3 \pm 2.7$ & $-7.6 \pm 3.6$ \\
& $\mathrm{c}$ & $4.6 \pm 0.6$ & $68.1 \pm 3.6$ & $-1.1 \pm 5.3$ & $77.9 \pm 2.3$ & $3.9 \pm 3.1$ \\
\multirow{2}{*}{ Spring } & $\mathrm{d}$ & $4.2 \pm 0.9$ & $10.5 \pm 1.0$ & $3.8 \pm 9.4$ & $48.6 \pm 3.2$ & $1.4 \pm 6.7$ \\
\hline \hline
\end{tabular}

c. These mean inclinations were all significantly different from each other (one-way ANOVA with post hoc multiple comparison, $\alpha=0.05$ ), indicating that the movement conditions a to $\mathrm{c}$ were indeed implemented with different rotations as planned. The movement durations were significantly different between condition $\mathrm{c}$ and conditions $\mathrm{a}$ and $\mathrm{b}$. The movement durations under conditions $\mathrm{a}$ and $\mathrm{b}$ were not significantly different from each other (one-way ANOVA with post hoc multiple comparison, $\alpha=0.05)$.

A summary of the estimated work and change of height for the mass and spring loads is given in Table II. The work performed was estimated on the basis of kinetic and kinematic sensor information (1), (3)-(8). The change of height was estimated using the kinematic sensors only (3)-(7). The error in the estimated work was calculated by taking the difference with the actual potential energy change derived from the independently measured height change. The error in the estimated height change derived from kinematic sensor information was calculated by comparison with the actual height change. We answered the question whether there were differences in accuracy between the estimates of performed work and height change (columns 5 and 7 of Table II) by testing the hypothesis that both estimates resulted in the same percentage error ( $\mathrm{t}=$ test, $\alpha=0.05)$. This hypothesis was rejected for movement conditions $\mathrm{a}, \mathrm{b}$, and $\mathrm{c}$ (mass load) but not for condition d (spring load). For the mass movements, the percentage error of estimated work was not significantly different between the three movement conditions $\mathrm{a}, \mathrm{b}$, and $\mathrm{c}$, while the percentage error of the estimated height change was significantly different between all three conditions (oneway ANOVA with post hoc multiple comparison, $\alpha=0.05$ ).

\section{DISCUSSION}

We have demonstrated the proposed concept of sensing power transfer for relatively short free movements using kinematic and kinetic sensors on a handle between the human body and the environment. The estimated performed work was accurate within $4 \%$ for varying movements with net displacements and varying loads (mass and spring).

In the tested loads, the height change could be estimated both from kinematic information and the work performed, since the loads were conservative. It should be noted that in many potential applications of power sensing, the loads are unknown or may have dissipative components, which may not allow for estimation of height or length change from performed work. In these cases, displacement is to be estimated from the kinematic sensor information only, and performed work can only be estimated by integrating estimated power transfer.

We have to consider that the estimation accuracy for displacement as well as power transfer and performed work may depend strongly on the complexity and duration of the movements. In our experiments, the error in the estimated work did not differ significantly between movement conditions a-c despite the fact that these movement conditions corresponded to different movement types (condition a: minimal rotation, b: pendulumlike movements, c: tilted mass), associated with different mean inclinations and different durations of the movements. The error in the estimate of the height change, in contrast, was significantly different between the three movement conditions. This may be due to the fact that estimation of the scalar quantity power by the nonlinear inner product operation (1) is only sensitive to errors of the velocity component in the direction of force, while the complete error in the vector quantity velocity influences the estimation of position change, of which subsequently only the $z$-component is used as the estimate of height change. Especially, under condition b, relatively long movement trajectories were realized with constantly changing orientation. This induces larger errors in the estimation of position change than under conditions a and c, which corresponded to considerably shorter movement trajectories and minimal or noncontinuous change of orientation.

The movements evaluated in our experiments all had a relatively short duration of several seconds (see Table II). The error in estimated work and height change is expected to increase for longer lasting movements, due to integration errors. Fortunately, human interactions with the environment are in many cases relatively short or have a cyclical nature. A priori information about the movement can be taken into account to improve the estimates of displacement and power transfer. In the current study, we have used initial and final zero velocity (6), (7) and initial orientation (3). If final orientation would be used as an additional constraint, the orientation and position estimates would further improve. However, the application of additional constraints restricts the applicability to more specific cases and errors are made if the constraints are not satisfied in actual movements.

The errors of the performed work and height changes were relatively low despite the relatively large range of the force sensors used: the ATI mini sensors had a range of $\pm 580 \mathrm{~N}$ in $x$ - and $y$-directions and $\pm 1160 \mathrm{~N}$ in the axial $z$-direction, while the forces required to move the $9.37 \mathrm{~kg}$ mass load were below $200 \mathrm{~N}$ and for the spring even below $50 \mathrm{~N}$ [Fig. 2(a) and (b)]. The sensed force, and therefore estimated power and 
work, could have been more accurate if force sensors were used with a smaller range in the order of the expected forces: in the current study, the offset force before and after the mass movement trials (unloaded handle) differed $4.5 \pm 1.6 \mathrm{~N}$ for condition a, $2.3 \pm 1.6 \mathrm{~N}$ for condition $\mathrm{b}$, and $0.5 \pm 0.5 \mathrm{~N}$ for condition c. Larger relative errors in the force exerted in the spring movements as compared to the mass movements may be the reason for the larger percentage error in the estimated work for the spring (movement condition d) than for mass movements, while the movement duration under condition $d$ was comparable to condition $\mathrm{c}$.

For the performed movements, the rotational power transfer was considerably smaller than the translational power (see Table I) and can, therefore, be neglected. When measuring at the interface between body and environment, the translational power transfer is always expected to be dominant. Even when generating torques, for example, by the hand, the effort is transferred to the environment by interface forces. Such sensing systems at the body surface consequently do not need to incorporate 3-D moment sensing. 3-D angular velocity sensing is, however, desirable, since it can improve the orientation estimation, which is an essential prerequisite for accurate estimation of acceleration. Estimation of acceleration requires accurate subtraction of gravitational acceleration from the accelerometer signals (5). For this purpose, the orientation of gravitational acceleration in sensor coordinates need to be known accurately at any time. It should be noted that the angular velocity may not need to be sensed on many places dispersed over the contact area of a hand or foot but merely at a single position, assuming that the angular velocity is approximately the same for the whole contact area.

The experimental setup used in the current study does not apply kinematic and kinetic sensors at the interface between body and environment, since it was merely meant to demonstrate the principle of power transfer sensing during free motions. However, kinematic and kinetic sensing at the surface of the body is potentially feasible when using matrices of miniature sensing systems of 3-D force and acceleration, spread over the contact area of body and load [48]. Power is transferred in most cases via the hands or feet but also sometimes via the trunk, pelvis, and thighs. It should be noted that for the purpose of estimating power transfer to the environment, only those body areas need to be measured that experience nonzero interaction forces and have a nonzero velocity in global coordinates. If the body moves with respect to an environment that moves at a constant speed, for example during cycling, the global coordinate system may of course be chosen fixed to that environment.

Apart from using measured force, moment, velocity, and angular velocity for the estimation of power transfer between body and environment (1), these quantities also provide information about the dynamic characteristics of the load as well as of the human body. The dynamics is in fact defined as the relation between both effort and flow variables, respectively, force and velocity. In general, it will not be possible to identify the dynamics of both bodies in their complete working range, since they are physically coupled during the interaction and, therefore, have a joint dynamics. Consequently, the joint movements and interaction forces have to satisfy the dynamics of each of both bodies and both bodies will not be persistently excited in their full individual working ranges, which is a prerequisite for adequate identification. Still, the relation between interaction force and movement can result in useful characterization of the dynamics of one or both bodies. For example, the relation between interaction force and movement can indicate whether either or both of the bodies mainly appear to be of inertial, elastic, or dissipative nature in the range of use. If any of these natures is dominant, the approximate apparent mass, stiffness, or damping can also be derived.

In addition to the analysis of mechanical performance in ergonomics and sports, the ability of the human neuromuscular system to adapt to the large variety of loads encountered in daily life may be assessed from the measured interaction forces and movements. Under healthy condition, humans can adapt their reflexive motor control flexibly and optimally to such varying loads, but patients with central neural disorders like stroke are not able to do so [49].

\section{ACKNOWLEDGMENT}

The technical support of Mr. E. Droog in realizing the experimental setup is gratefully acknowledged.

\section{REFERENCES}

[1] M. A. Huysmans, M. J. Hoozemans, B. Visser, and J. H. van Dieen, "Grip force control in patients with neck and upper extremity pain and healthy controls," Clin. Neurophysiol., vol. 119, pp. 1840-1848, 2008.

[2] I. Kingma, T. Bosch, L. Bruins, and J. H. van Dieen, "Foot positioning instruction, initial vertical load position and lifting technique: Effects on low back loading," Ergonomics, vol. 47, pp. 1365-1385, 2004.

[3] I. Kingma, J. H. van Dieen, M. de Looze, H. M. Toussaint, P. Dolan, and C. T. Baten, "Asymmetric low back loading in asymmetric lifting movements is not prevented by pelvic twist," J. Biomech., vol. 31, pp. 527-534, 1998.

[4] R. Colombo, F. Pisano, S. Micera, A. Mazzone, C. Delconte, M. C. Carrozza, P. Dario, and G. Minuco, "Assessing mechanisms of recovery during robot-aided neurorehabilitation of the upper limb," Neurorehabil. Neural Repair, vol. 22, pp. 50-63, 2008.

[5] G. Kwakkel, B. J. Kollen, and H. I. Krebs, "Effects of robot-assisted therapy on upper limb recovery after stroke: a systematic review," Neurorehabil. Neural Repair, vol. 22, pp. 111-121, 2008.

[6] B. T. Volpe, D. Lynch, A. Rykman-Berland, M. Ferraro, M. Galgano, N. Hogan, and H. I. Krebs, "Intensive sensorimotor arm training mediated by therapist or robot improves hemiparesis in patients with chronic stroke," Neurorehabil. Neural Repair, vol. 22, pp. 305-310, 2008.

[7] F. Hintzy and N. Tordi, "Mechanical efficiency during hand-rim wheelchair propulsion: effects of base-line subtraction and power output," Clin. Biomech. (Bristol, Avon), vol. 19, pp. 343-349, 2004.

[8] L. H. Van Der Woude, A. Horstman, P. Faas, S. Mechielsen, H. A. Bafghi, and J. J. de Koning, "Power output and metabolic cost of synchronous and asynchronous submaximal and peak level hand cycling on a motor driven treadmill in able-bodied male subjects," Med. Eng. Phys., vol. 30, pp. 574-580, 2008.

[9] E. A. Hansen and H. Waldeland, "Seated versus standing position for maximization of performance during intense uphill cycling," J. Sports Sci., vol. 26, pp. 977-984, 2008.

[10] M. J. Hofmijster, A. J. Van Soest, and J. J. De Koning, "Rowing skill affects power loss on a modified rowing ergometer," Med. Sci. Sports Exerc., vol. 40, pp. 1101-1110, 2008.

[11] S. A. Ingham, G. P. Whyte, K. Jones, and A. M. Nevill, "Determinants of 2,000 m rowing ergometer performance in elite rowers," Eur. J. Appl. Physiol., vol. 88, pp. 243-246, 2002.

[12] H. I. Krebs, J. J. Palazzolo, L. Dipietro, M. Ferraro, J. Krol, K. Rannekleiv, B. T. Volpe, and N. Hogan, "Rehabilitation robotics: Performance-based progressive robot-assisted therapy," Auton. Robots, vol. 15, pp. 7-20, 2003. 
[13] R. Loureiro, F. Amirabdollahian, M. Topping, B. Driessen, and W. Harwin, "Upper limb robot mediated stroke teherapy - GENTLE/s approach," Auton. Robots, vol. 15, pp. 35-51, 2003.

[14] S. Micera, M. C. Carroza, E. Guglielmeelli, G. Cappiello, F. Zaccone, C. Freschi, R. Colombo, A. Mazzone, C. Delconte, F. Pisano, G. Minuco, and P. Dario, "A simple robotic system for neurorehabilitation," Auton. Robots, vol. 19, pp. 271-284, 2005.

[15] J. F. Veneman, R. Kruidhof, E. E. Hekman, R. Ekkelenkamp, E. H. Van Asseldonk, and H. Van Der Kooij, "Design and evaluation of the LOPES exoskeleton robot for interactive gait rehabilitation," IEEE Trans. Neural Syst. Rehabil. Eng., vol. 15, no. 3, pp. 379-386, Sep. 2007.

[16] H. J. Hermens and M. M. Vollenbroek-Hutten, "Towards remote monitoring and remotely supervised training," J. Electromyogr. Kinesiol., vol. 18, pp. 908-919, 2008.

[17] D. A. Commissaris and H. M. Toussaint, "Load knowledge affects lowback loading and control of balance in lifting tasks," Ergonomics, vol. 40, pp. 559-575, 1997.

[18] J. H. Skotte, M. Essendrop, A. F. Hansen, and B. Schibye, "A dynamic 3D biomechanical evaluation of the load on the low back during different patient-handling tasks," J. Biomech., vol. 35, pp. 1357-1366, 2002.

[19] I. Kingma, C. T. Baten, P. Dolan, H. M. Toussaint, J. H. van Dieen, M. P. de Looze, and M. A. Adams, "Lumbar loading during lifting: a comparative study of three measurement techniques," J. Electromyogr. Kinesiol., vol. 11, pp. 337-345, 2001.

[20] R. Colombo, F. Pisano, S. Micera, A. Mazzone, C. Delconte, M. C. Carrozza, P. Dario, and G. Minuco, "Robotic techniques for upper limb evaluation and rehabilitation of stroke patients," IEEE Trans. Neural Syst. Rehabil. Eng., vol. 13, no. 3, pp. 311-324, Sep. 2005.

[21] H. I. Krebs, S. Mernoff, S. E. Fasoli, R. Hughes, J. Stein, and N. Hogan, "A comparison of functional and impairment-based robotic training in severe to moderate chronic stroke: A pilot study," NeuroRehabilitation, vol. 23, pp. 81-87, 2008.

[22] P. C. Breedveld, "Port-based modeling of mechatronics systems," Math. Comput. Simul., vol. 66, pp. 99-127, 2004.

[23] A. Forner-Cordero, H. J. Koopman, and F. C. Van Der Helm, "Inverse dynamics calculations during gait with restricted ground reaction force information from pressure insoles," Gait Posture, vol. 23, pp. 189-199, 2006.

[24] J. R. Mackey and B. L. Davis, "Simultaneous shear and pressure sensor array for assessing pressure and shear at foot/ground interface," $J$. Biomech., vol. 39, pp. 2893-2897, 2006.

[25] M. A. Razian and M. G. Pepper, "Design, development, and characteristics of an in-shoe triaxial pressure measurement transducer utilizing a single element of piezoelectric copolymer film," IEEE Trans. Neural Syst. Rehabil. Eng., vol. 11, no. 3, pp. 288-293, Sep. 2003.

[26] K. Aminian, B. Najafi, C. Bula, P. F. Leyvraz, and P. Robert, "Spatiotemporal parameters of gait measured by an ambulatory system using miniature gyroscopes," J. Biomech., vol. 35, pp. 689-699, 2002.

[27] K. Aminian, K. Rezakhanlou, E. De Andres, C. Fritsch, P. F. Leyvraz, and P. Robert, "Temporal feature estimation during walking using miniature accelerometers: An analysis of gait improvement after hip arthroplasty," Med. Biol. Eng. Comput., vol. 37, pp. 686-691, 1999.

[28] E. B. Bachmann, "Inertial and magnetic tracking of limb segment orientation for inserting humans into synthetic environments," Ph.D. thesis, Naval Postgraduate School, Monterey, CA, 2000.

[29] F. Ferraris, U. Grimalde, and M. Parvis, "Procedure for effortless in-field calibration of three-axis rate gyros and accelerometers," Sens. Mater. vol. 7, pp. 311-330, 1995.

[30] E. Foxlin, "Inertial head-tracker sensor fusion by a complementary separate-bias Kalman filter," in Proc. VRAIS 1996, pp. 185-194.

[31] B. Kemp, A. J. Janssen, and B. van Der Kamp, "Body position can be monitored in 3D using miniature accelerometers and earth-magnetic field sensors," Electroencephalogr. Clin. Neurophysiol., vol. 109, pp. 484-488, 1998.

[32] J. C. Lötters, J. Schipper, P. H. Veltink, W. Olthuis, and P. Bergveld, "Procedure for in-use calibration of triaxial accelerometers in medical applications," Sens. Actuators A, vol. 68, pp. 221-228, 1998.

[33] H. J. Luinge and P. H. Veltink, "Inclination measurement of human movement using a 3-D accelerometer with autocalibration," IEEE Trans. Neural Syst. Rehabil. Eng., vol. 12, no. 1, pp. 112-121, Mar. 2004.

[34] H. J. Luinge and P. H. Veltink, "Measuring orientation of human body segments using miniature gyroscopes and accelerometers," Med. Biol. Eng. Comput., vol. 43, pp. 273-282, 2005.

[35] H. J. Luinge, P. H. Veltink, and C. T. Baten, "Ambulatory measurement of arm orientation,” J. Biomech., vol. 40, pp. 78-85, 2007.
[36] R. E. Mayagoitia, A. V. Nene, and P. H. Veltink, "Accelerometer and rate gyroscope measurement of kinematics: An inexpensive alternative to optical motion analysis systems," J. Biomech., vol. 35, pp. 537-542, 2002.

[37] J. R. W. Morris, "Accelerometry-A technique for the measurement of human body movements," J. Biomech., vol. 6, pp. 729-736, 1973.

[38] D. Roetenberg, C. T. Baten, and P. H. Veltink, "Estimating body segment orientation by applying inertial and magnetic sensing near ferromagnetic materials," IEEE Trans. Neural Syst. Rehabil. Eng., vol. 15, no. 3, pp. 469471, Sep. 2007.

[39] A. Salarian, H. Russmann, C. Wider, P. R. Burkhard, F. J. Vingerhoets, and K. Aminian, "Quantification of tremor and bradykinesia in Parkinson's disease using a novel ambulatory monitoring system," IEEE Trans. Biomed. Eng., vol. 54, no. 2, pp. 313-322, Feb. 2007.

[40] P. H. Veltink, P. Slycke, J. Hemssems, R. Buschman, G. Bultstra, and H. Hermens, "Three dimensional inertial sensing of foot movements for automatic tuning of a two-channel implantable drop-foot stimulator," Med. Eng. Phys., vol. 25, pp. 21-28, 2003.

[41] A. T. Willemsen, J. A. van Alste, and H. B. Boom, "Real-time gait assessment utilizing a new way of accelerometry," J. Biomech., vol. 23 , pp. 859-863, 1990.

[42] L. Williamson and B. J. Andrews, "Detecting absolute human knee angle and angular velocity using accelerometers and rate gyroscopes," Med. Biol. Eng. Comput., vol. 39, pp. 65-75, 2003.

[43] H. M. Schepers, H. F. Koopman, and P. H. Veltink, "Ambulatory assessment of ankle and foot dynamics," IEEE Trans. Biomed. Eng., vol. 54, no. 5, pp. 895-902, May 2007.

[44] P. H. Veltink, C. Liedtke, E. Droog, and H. van Der Kooij, "Ambulatory measurement of ground reaction forces," IEEE Trans. Neural Syst. Rehabil. Eng., vol. 13, no. 3, pp. 423-427, Sep. 2005.

[45] P. H. Veltink, H. G. Kortier, and H. M. Schepers, "Sensing dynamic interaction with the environment," in Proc. Int. Conf. Ambul. Monit. Phys. Activity Movement, Rotterdam, 2008, p. 167.

[46] D. Roetenberg, Inertial and Magnetic Sensing of Human Motion. Enschede, The Netherlands: University of Twente, 2006.

[47] J. E. Bortz, "A new mathematical formulation for strapdown inertial navigation," IEEE Trans. Aerosp. Electron. Syst., vol. AES-7, no. 1, pp. 61-66, Jan. 1971.

[48] P. H. Veltink, "Device and method for measuring the dynamic interaction between bodies," Patent Application 2000835, The Netherlands, 2007.

[49] A. C. Schouten, W. J. Van de Beek, J. J. Van Hilten, and F. C. Van Der Helm, "Proprioceptive reflexes in patients with reflex sympathetic dystrophy," Exp. Brain Res., vol. 151, pp. 1-8, 2003.

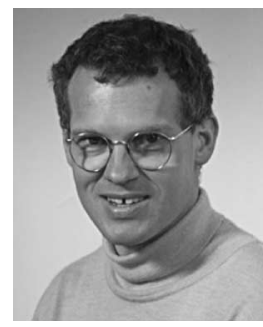

Peter H. Veltink (S'85-M'88-SM'06) was born in Groenlo, The Netherlands, in 1960. He received the M.S degree (cum laude) in electrical engineering and the $\mathrm{Ph} . \mathrm{D}$. degree in electrical nerve stimulation from the University of Twente, Enschede, The Netherlands, in 1984 and 1988, respectively.

He performed sabbaticals at Case Western Reserve University, Cleveland, in 1989, and at the Center for Sensory-Motor-Interaction at Aalborg University, Denmark, in 1997. He is currently a Professor of technology for the restoration of human function and the Chairman of the Biomedical Signals and Systems Department, University of Twente, Institute for Biomedical Technology (BMTI). His current research interests include neural stimulation and sensing, artificial human motor control, and human movement sensing. He has been a Scientific Coordinator of 3 European Union (EU) research training networks and leads or is involved in various projects financed by the EU, the Dutch Ministry of Economic Affairs, and the Dutch Foundation for Technical Sciences School-to-Work (STW).

Prof. Veltink was the Treasurer of the International Functional Electrical Stimulation Society (IFESS) from 1996 to 2001. Since 2005, he has been the Chair of the Benelux IEEE-Engineering in Medicine and Biology Society (EMBS) chapter. He received the Royal Shell Stimulating Prize in 1997 for his contribution to the rehabilitation-engineering field. 


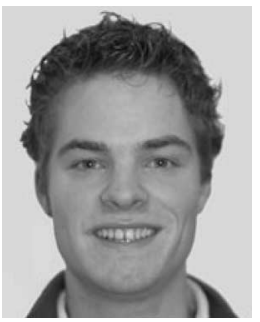

Henk Kortier was born in Hengelo (OV), The Netherlands, on November 13, 1984. He received the B.Sc. degree in electrical engineering in 2008 from the University of Twente, Enschede, The Netherlands, where he is currently working toward the M.Sc. degree.

He was engaged in sensing power transfer between human and environment.

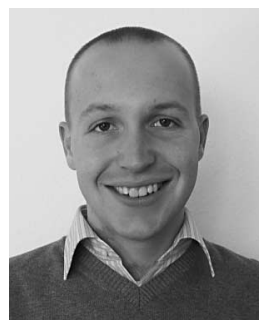

H. Martin Schepers (S'06) was born in Hardenberg, The Netherlands, on July 4, 1981. He received the M.Sc. degree in electrical engineering in 2004 from the University of Twente, Enschede, The Netherlands, he is currently working toward the Ph.D. degree.

His current research interests include ambulatory sensor systems, movement analysis, and biomedical signal processing. 